\title{
Strategies and limits in multi-stage single-point incremental forming
}

\author{
Skjødt, Martin; Silva, M.B.; Martins, P. A. F.; Bay, Niels
}

Published in:

Journal of Strain Analysis for Engineering Design

Link to article, DOI:

10.1243/03093247JSA574

Publication date:

2010

Document Version

Publisher's PDF, also known as Version of record

Link back to DTU Orbit

Citation (APA):

Skjødt, M., Silva, M. B., Martins, P. A. F., \& Bay, N. (2010). Strategies and limits in multi-stage single-point incremental forming. Journal of Strain Analysis for Engineering Design, 45(1), 33-44.

https://doi.org/10.1243/03093247JSA574

\section{General rights}

Copyright and moral rights for the publications made accessible in the public portal are retained by the authors and/or other copyright owners and it is a condition of accessing publications that users recognise and abide by the legal requirements associated with these rights.

- Users may download and print one copy of any publication from the public portal for the purpose of private study or research.

- You may not further distribute the material or use it for any profit-making activity or commercial gain

- You may freely distribute the URL identifying the publication in the public portal

If you believe that this document breaches copyright please contact us providing details, and we will remove access to the work immediately and investigate your claim 


\title{
Strategies and limits in multi-stage single-point incremental forming
}

\author{
M Skjoedt $^{1}$, M B Silva ${ }^{2}$, P A F Martins ${ }^{2 *}$, and N Bay ${ }^{1}$ \\ ${ }^{1}$ Technical University of Denmark, Department of Mechanical Engineering, Kongens Lyngby, Denmark \\ ${ }^{2}$ IDMEC, Instituto Superior Tecnico, Universidade Técnica de Lisboa, Lisboa, Portugal
}

The manuscript was received on 28 June 2009 and was accepted after revision for publication on 27 August 2009.

DOI: 10.1243/03093247JSA574

\begin{abstract}
Multi-stage single-point incremental forming (SPIF) is a state-of-the-art manufacturing process that allows small-quantity production of complex sheet metal parts with vertical walls. This paper is focused on the application of multi-stage SPIF with the objective of producing cylindrical cups with vertical walls. The strategy consists of forming a conical cup with a taper angle in the first stage, followed by three subsequent stages that progressively move the conical shape towards the desired cylindrical geometry.

The investigation includes material characterization, determination of forming-limit curves and fracture forming-limit curves (FFLCs), numerical simulation, and experimentation, namely the evaluation of strain paths and fracture strains in actual multi-stage parts.

Assessment of numerical simulation with experimentation shows good agreement between computed and measured strain and strain paths. The results also reveal that the sequence of multi-stage forming has a large effect on the location of strain points in the principal strain space. Strain paths are linear in the first stage and highly non-linear in the subsequent forming stages. The overall results show that the experimentally determined FFLCs can successfully be employed to establish the forming limits of multi-stage SPIF.
\end{abstract}

Keywords: single-point incremental forming, multi-stage forming, forming-limit curve, fracture forming-limit curve

\section{INTRODUCTION}

Single-point incremental forming (SPIF) is a new sheet-metal-forming process with a high-potential economic pay-off for rapid prototyping applications and for small-quantity production.

The basic components in the SPIF process are presented in Fig. 1: sheet metal blank, blank holder, backing plate, and rotating single-point forming tool. The blank holder is utilized to clamp and hold the blank sheet in position during the SPIF process. The backing plate supports the sheet, and its opening defines the working area of the single-point forming tool. The tool is utilized to shape the sheet progressively into a component and its path is

*Corresponding author: Departamento de Engenharia Mecanica, Instituto Superior Tecnico, Seccao de Tecnologia Mecanica, Avenida Rovisco Pais, Lisboa 1049-001, Portugal.

email:pmartins@ist.utl.pt generated by a computer numerical control (CNC) machining centre. During the forming process there is no backup die supporting the back surface of the sheet.

The main advantages of SPIF over the conventional sheet-metal-forming process are as follows:

(a) the increase in material formability due to the incremental nature of the process;

(b) the great flexibility derived from the absence of positive or negative dies;

(c) the advantage of utilizing a conventional $\mathrm{CNC}$ milling machine;

(d) the possibility that design changes can be easily and quickly evaluated using computer aided design and computer aided manufacture (CAM) data to produce the parts directly [1].

In the last few years the governing mode of deformation in SPIF has been the subject of controversy in the metal-forming community [2]. 


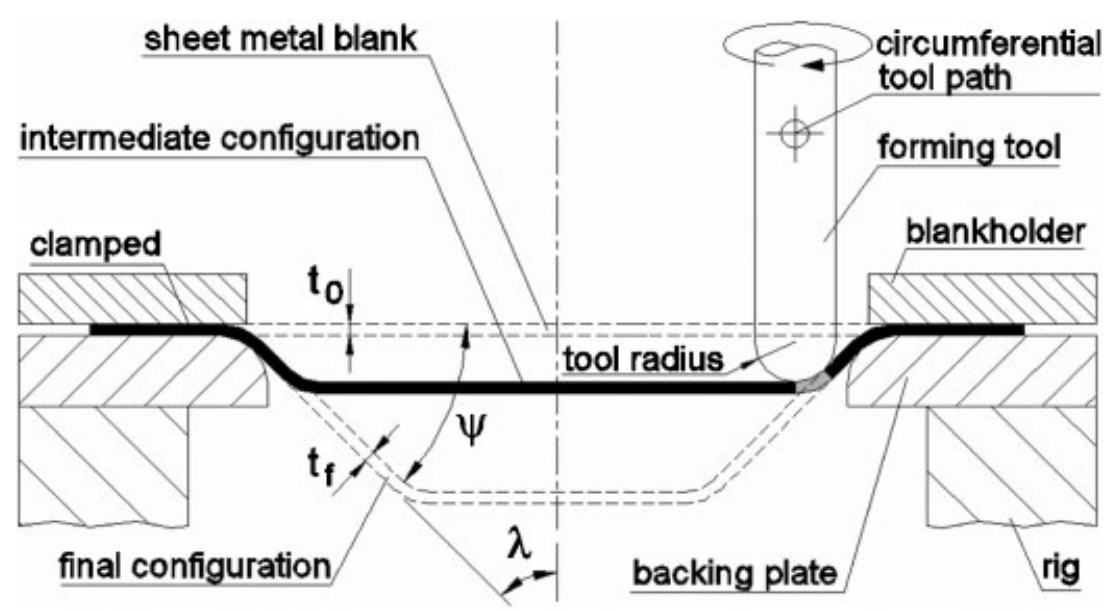

Fig. 1 Schematic representation of a cross-sectional view of the rotational symmetric (SPIF) process

Some researchers have claimed that deformation takes place by stretching instead of shearing while others have claimed the opposite, but assertions are mainly based on 'similarities' to well-known processes of stamping and shear spinning rather than on experimental evidence from SPIF itself. In previous work [3], the present authors presented a theoretical framework built upon the combined utilization of membrane analysis and ductile damage mechanics that is capable of addressing the fundamentals of SPIF of metallic sheets. The theoretical framework accounts for the influence of major process parameters and allows their mutual interaction to be studied both qualitatively and quantitatively. It also allows the conclusion that the likely mode of material failure in SPIF is consistent with stretching, rather than shearing being the governing mode of deformation. More recently, numerical simulations combined with the experimental observation of the suppression of neck formation and the study of the morphology of the cracks enabled the present authors to conclude that traditional forming-limit curves (FLCs) are inapplicable to describe failure. Instead, fracture forminglimit curves (FFLCs) should be employed to evaluate the overall formability of the process [4].

Plastic deformation in SPIF takes place by uniform thinning until fracture without experimental evidence of localized necking before reaching the onset of fracture [3]. The production by SPIF of conventional (i.e. single-stage) parts with shapes having straight vertical walls is impossible since, according to the sine law, a $90^{\circ}$ drawing angle would lead to a wall thicknesses equal to zero and strains towards infinity.

This paper deals with new concepts of multi-stage SPIF that have been recently proposed for eliminat- ing the aforementioned drawback of conventional SPIF related to the production of complex sheet metal parts with vertical walls. The first attempts, as far as the present authors are aware, to utilize multistage SPIF were made by Kitazawa et al. [5] and Kitazawa and Nakane [6] who produced hemiellipsoidal axisymmetric parts by employing two sequential stages. The first stage was utilized to shape an intermediate conical geometry that enabled these workers to form the desired hemiellipsoidal shape during the second stage. Later, Kim and Yang [7] and Young and Jeswiet [8] utilized two-stage forming sequences with the purpose of achieving a more uniform distribution of thickness in the parts and concluded that multi-stage strategies significantly improve the overall formability of the process, allowing the production of parts that could not be obtained by conventional SPIF.

The extension of multi-stage forming methodologies to variants of incremental sheet metal forming was also attempted by other researchers. For instance, Hirt and co-workers $[\mathbf{9}, \mathbf{1 0}]$ proposed a multi-stage two-point incremental forming methodology for producing non-axisymmetric sheet metal parts. The approach consists of alternating the tool movement from upwards to downwards, and from stage to stage the angle is increased. The number of intermediate stages is chosen to be as small as possible in order to avoid surface wear and to limit the overall forming time. The risk of wrinkling is said to increase with the decrease in the intermediate number of stages.

Very recently Skjoedt $e t$ al. $[\mathbf{1 1}]$ and Verbert $e t$ al. [12] defined new multi-stage forming strategies to produce SPIF parts with vertical walls. Skjoedt et al. [11] reported the production of a cylindrical cup, 
starting from a cone with a $45^{\circ}$ angle, and progressively moving the middle of the conical wall towards the corner of the desired cylindrical shape. Verbert $e t$ al. [12] presented a cylindrical part that was successfully produced in five steps, starting from a cone angle of $50^{\circ}$ and increasing its angle by $10^{\circ}$ in each subsequent step. They also showed complex parts produced by means of automatic multi-stage tool path generation but provided no details on the number of stages and forming sequences. Finally, Duflou et al. [13] studied formability in multi-stage tool paths and concluded that process windows are extended as a consequence of the straining of (semi)horizontal areas of the parts that remain unaffected in conventional tool path strategies.

The aim of the present paper is twofold: first, to check whether FFLCs can be successfully employed to establish the forming limits of multi-stage SPIF and, second, to present an enhanced multi-stage SPIF sequence that allows the production of flatbottom cylindrical cups with vertical walls. The presentation covers both numerical simulation and experimentation research topics. The numerical modelling was performed using the explicit-dynamic finite element computer program LS-DYNA. The experimental work involved material characterization, determination of FLCs and FFLCs, and measurement and calculation of strain paths and fracture strains in actual SPIF parts.

\section{EXPERIMENTAL WORK}

The experimental work was performed on aluminium alloy AA1050-O sheet blanks of $1 \mathrm{~mm}$ thickness. Tensile and hydraulic bulge tests were utilized for performing the mechanical and formability characterization of the material. Multi-stage SPIF experiments were performed in order to produce parts with vertical walls and to support the overall numerical and experimental investigation.

\subsection{Mechanical characterization and formability limits}

The mechanical and formability characterization of the AA1050-O sheet blanks made use of tensile tests and biaxial, circular (diameter, $100 \mathrm{~mm}$ ), and elliptical (diameters of the major and minor axes, $100 \mathrm{~mm}$ and $63 \mathrm{~mm}$ respectively) hydraulic bulge tests (Fig. 2). The tensile tests were performed in a universal materials-testing machine (Instron 4507), in accordance with the standard for tensile tests NP

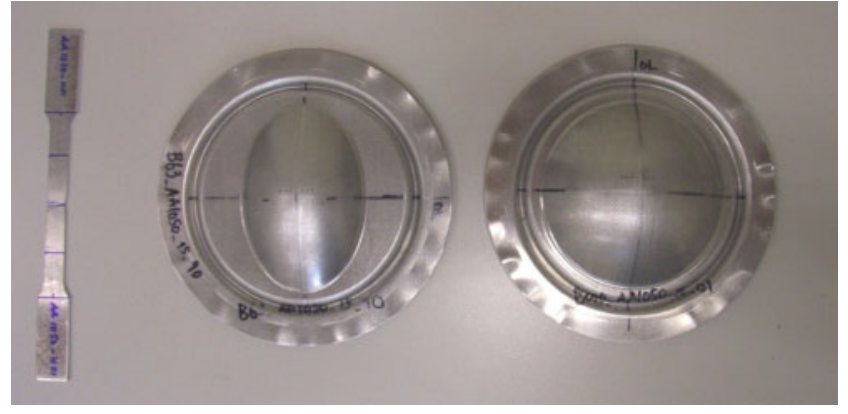

Fig. 2 Tensile, elliptical, and circular hydraulic bulge specimens utilized in the characterization of the formability limits of aluminium alloy AA1050-O

EN 10002-1 [14] and the hydraulic bulge tests were performed in a universal sheet-metal-testing machine (Erichsen 145/60).

The results obtained for the tensile tests are presented in Table 1. The symbol $E$ denotes Young's modulus, $\sigma_{\mathrm{y}}$ is the yield strength, $A$ is the uniform elongation at break point, and $U_{\mathrm{T}}$ is the toughness.

The stress-strain curve of aluminium alloy AA1050-O derived from the tensile tests is given by

$$
\bar{\sigma}=153 \bar{\varepsilon}^{0.25} \mathrm{MPa}
$$

The experimental technique utilized for obtaining the FLC involved electrochemical etching a grid of circles of $2 \mathrm{~mm}$ initial diameter on the surface of the sheets before forming and measuring the major and minor axes of the ellipses that result from the plastic deformation in the formability tests. The FLC was estimated by taking the strains $\left(\varepsilon_{1}, \varepsilon_{2}\right)$ at failure from grid elements placed just outside the neck (i.e. adjacent to the region of intense localization) since they represent the condition of the uniformly thinned sheet just before necking occurs $[\mathbf{1 5}]$. The resulting FLC is plotted in Fig. 3 and its intersection with the major strain axis, which is found to occur at $\varepsilon_{1}=0.24$, is in good agreement with the value of the strain-hardening exponent of the stress-strain curve obtained by means of tensile tests (equation (1)).

The procedure for determination of the FFLC is different from that of the FLC because it requires measuring the thickness at fracture in order to

Table 1 Results from the tensile tests on AA1050-O sheets

\begin{tabular}{ll}
\hline Parameter (units) & Value \\
\hline$E(\mathrm{MPa})$ & 70943 \\
$\sigma_{\mathrm{y}}(\mathrm{MPa})$ & 119.9 \\
$U_{\mathrm{T}}(\mathrm{MPa})$ & 9.33 \\
$A(\%)$ & 10 \\
\hline
\end{tabular}




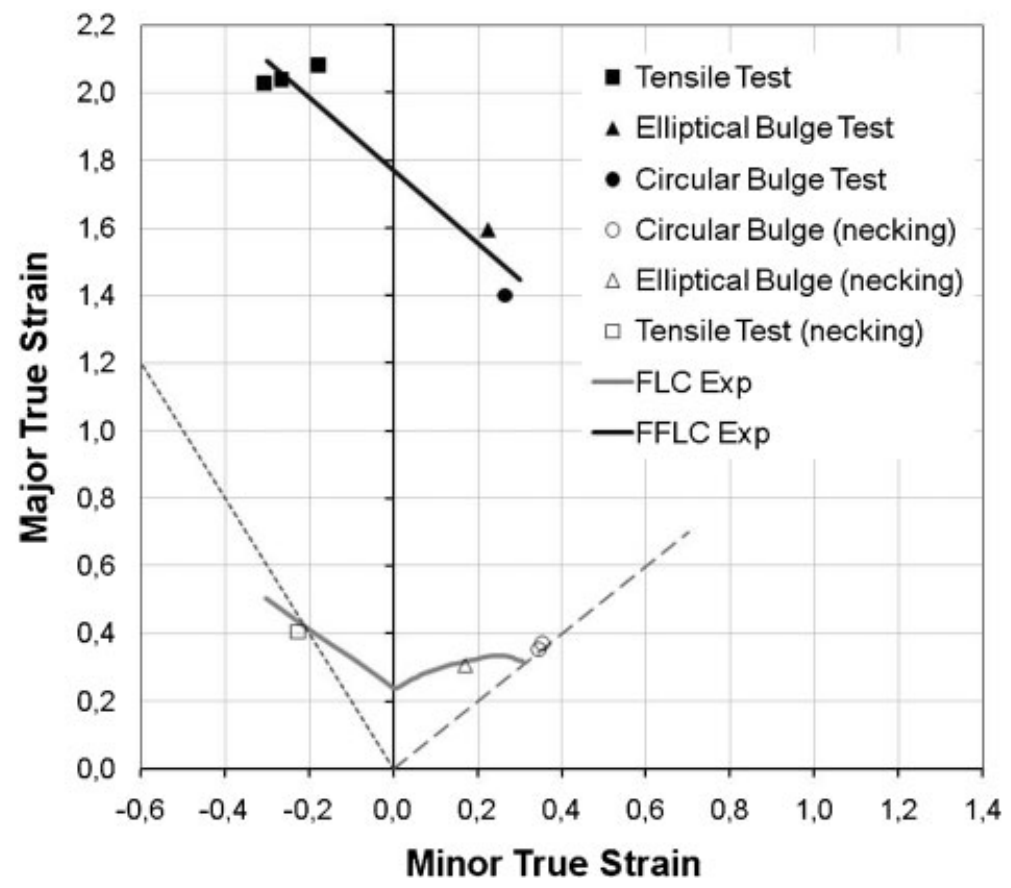

Fig. 3 FLC and FFLC for aluminium alloy AA1050-O sheets of $1 \mathrm{~mm}$ thickness

obtain the 'gauge length' strains. The adopted procedure involved measuring the length increase parallel to the crack using the grid technique in order to obtain the strain in this direction. The thickness strain was determined by measuring the sheet thickness using a microscope at several places along the crack, and the third fracture strain component, in the plane of the sheet and direction perpendicular to the crack, was determined by volume constancy, knowing the two other strains. The experimental FFLC is also plotted in Fig. 3 and can be approximated by a straight line $\varepsilon_{1}+1.08 \varepsilon_{2}=1.77$ falling from left to right, which is close to the condition of constant through-thickness strain at fracture (given by a slope of -1 ) [16].

The large distance between the neck formation FLC and the fracture FFLC in Fig. 3 indicates that AA1050-O is a very ductile material allowing a considerable through-thickness strain within the neck, between neck initiation and fracture.

\subsection{Multi-stage SPIF}

In previous work, Skjoedt et al. [11] presented an innovative multi-stage SPIF strategy that enables the production of a cup with a vertical wall by SPIF. The strategy makes use of the four intermediate stages that are schematically plotted in Fig. 4 in order to extend deformation to all the material available.

The first stage is utilized for forming the sheet blank into a conical shape with a constant angle of

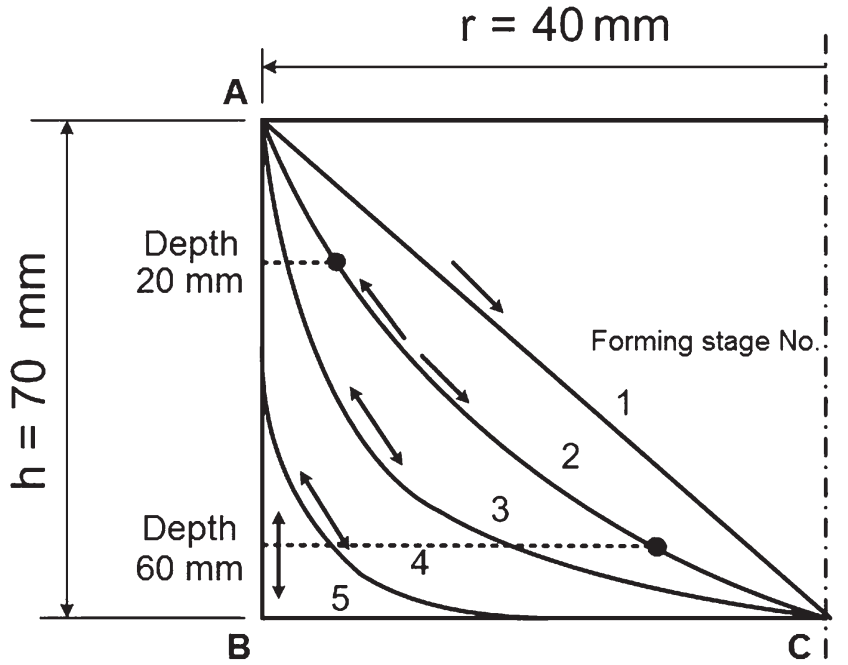

Fig. 4 Multi-stage SPIF strategy for producing a cylindrical cup with vertical walls [11]

$45^{\circ}$ while the subsequent stages are employed for gradually moving the middle of the conical wall towards the corner of the cylinder. All stages, apart from the first, can be performed with the tool moving either downwards or upwards. For convenience, when the movement of the tool is downwards the stage is represented by a $\mathrm{D}$, and when it is upwards by a $U$.

The fifth stage in Fig. 4 is supposed to produce an ideal final shape that is not technologically feasible because it would require the formatiion of a flatbottom cylindrical cup with a vertical wall and a 
bottom radius equal to zero. Still, assuming the extreme forming conditions of the fifth stage, the principal strains in the resulting SPIF part can be estimated by considering the deformation to be pure biaxial stretching and the meridional strain $\varepsilon_{\phi}$ to be evenly distributed. The circumferential strain $\varepsilon_{\theta}$ is zero at points $\mathrm{A}$ and $\mathrm{C}$ and maximum at point $\mathrm{B}$ (Fig. 4) and, in the case when $h=r$, the strain field is given by

$$
\begin{aligned}
& \varepsilon_{\phi}=\ln \left(\frac{2 r}{r}\right)=\ln (2) \\
& \varepsilon_{\theta, \max }=\ln \left(\frac{2 \pi r}{\pi r}\right)=\ln (2) \\
& \varepsilon_{t, \max }=-\ln (4) \approx-1.4
\end{aligned}
$$

Previous investigations by Skjoedt et al. [11, 17] revealed that a multi-stage SPIF strategy consisting of three downward movements followed by a final upward movement (DDDU) could be successfully employed to produce a sound cylindrical cup with vertical walls. This is the main reason why the overall investigation was focused on the analysis of multistage SPIF strategies that are exclusively based on four intermediate stages.

Multi-stage SPIF was performed in a Cincinnati Milacron Sabre 750 CNC machining centre equipped with appropriate experimental apparatus. The forming tool has a diameter of $12 \mathrm{~mm}$ and a hemispherical tip and was made of cold-worked tool steel (120WV4-DIN) hardened and tempered to a Rockwell $\mathrm{C}$ hardness of $60 \mathrm{HRC}$ in the working region. The feed rate was set to $1000 \mathrm{~mm} / \mathrm{min}$ and the first stage, which has a constant drawing angle, is performed with a vertical step size of $0.5 \mathrm{~mm}$. In subsequent stages it is not advantageous to utilize a fixed vertical step size because it results in a large distance between tool paths in flat regions where the drawing angle is close to zero, e.g. the bottom of the cup. Instead the general distance is set to $1 \mathrm{~mm}$, which results in a vertical step size of $1 \mathrm{~mm}$ in sections close to vertical and a vertical step size close to $0 \mathrm{~mm}$ in sections that are close to being flat.

Tool paths were generated with the program HeToPaC [18]. The lubricant applied between the forming tool and the sheet was diluted cutting fluid.

The geometries utilized in the intermediate SPIF stages were similar to those plotted in Fig. 4, with $h=70 \mathrm{~mm}$ and $r=80.5 \mathrm{~mm}$. The initial blanks consisted of square sheets of area $253 \mathrm{~mm} \times 253 \mathrm{~mm}$ that were electrochemically etched to imprint a grid with circles of $2 \mathrm{~mm}$ diameter on its surface to allow the principal strains to be measured after deformation.

\section{FINITE ELEMENT WORK}

The finite element model of the sheet blanks was built upon an initial course mesh of $26 \times 26$ shell elements, each having a side length about $9.7 \mathrm{~mm}$ (element type 16 in LS-DYNA). A full integration shell formulation was used with five integration points over the sheet thickness. Adaptive mesh refinement was utilized throughout the computation in order to limit the interference between the sheet and the contours of the forming tool and the backing plate, as well as to obtain high levels of accuracy in terms of geometry and distribution of field variables. The adaptive mesh refinement procedure consisted of three refinement operations ending up by splitting the original elements into 64 new elements that have one eighth of the initial element size.

The description of the forming tool and of the backing plate was performed by means of surface meshes. Both active tool components were considered rigid and a large number of elements were utilized to model its geometry in order to reduce the level of roughness that was artificially introduced by the overall discretization procedure. The movement of the tool in the finite element model was identical with that in the actual SPIF process, including the rotation and the helical path, which was defined by means of a large number of points. The number of points was determined by the tolerance setting in the CAM program.

Acceleration of the overall central processing unit (CPU) time was performed by means of a loadfactoring (or time-scaling) procedure. This changed the rate of loading by an artificial increase in the velocity of the single-point forming tool by a factor of 1500 for the first stage and a factor of 1000 in subsequent stages, in comparison with the real forming velocity. No mass scaling was used. The maximum increment of time step for performing the explicit central difference time integration scheme was based on a characteristic length equal to the shell area divided by the longest diagonal. As a precaution, LS-DYNA uses 0.9 times this value to guarantee stability. The material of the sheet was considered isotropic with the stress-strain curve presented in equation (1). No anisotropy effects were taken into consideration. 
The finite element simulation of each SPIF stage under the above-mentioned modelling conditions was computationally very intense. The full-scale model often required a CPU time of $120-240 \mathrm{~h}$ in a $900 \mathrm{MHz}$ computer.

\section{RESULTS AND DISCUSSION}

This section analyses the numerical and experimental strain paths resulting from multi-stage SPIF, provides an enhanced forming sequence that allows the production of flat-bottom cylindrical cups with vertical walls, and presents a comprehensive discussion on the validity of FFLCs in multi-stage SPIF.

\subsection{First stage}

Multi-stage SPIF is initiated by producing a cone with a constant drawing angle of $45^{\circ}$ in the downward movement of the forming tool. This results in evenly distributed strains along the sheet metal part except close to the backing plate and the bottom region of the cone where the radius is approaching the tool radius. Figure 5(a) presents numerical and experimental values of the major and minor strains over the surface of the parts. The agreement is very good and the strains are very close to plane strain, as would be expected from conventional SPIF of a conical shape. The finite element computed evolutions of the strain paths for two elements of the mesh model located at $10 \mathrm{~mm}$ and $60 \mathrm{~mm}$ depths at the end of the first stage are close to straight (Fig. 5(b)),

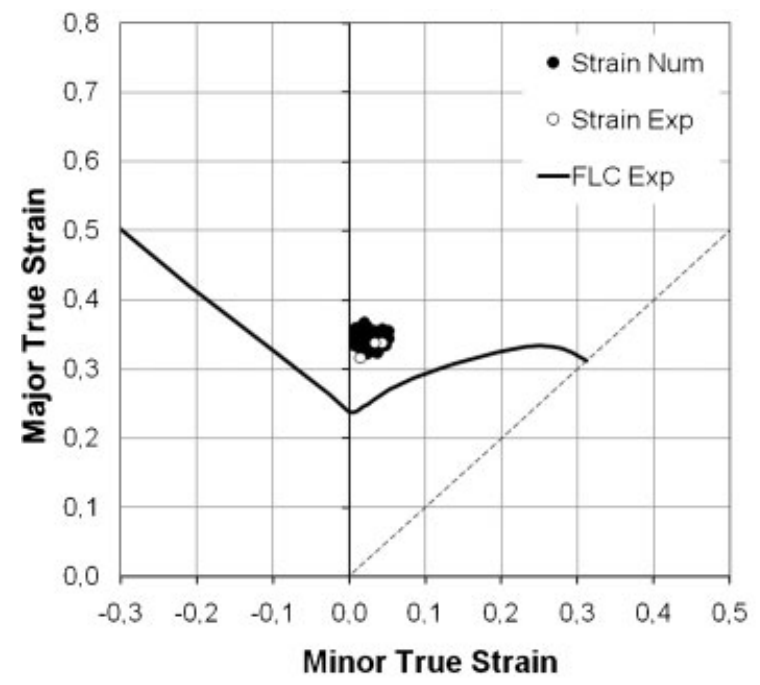

(a) and the serrated strain paths previously mentioned by other researchers were not observed.

The results also indicate that surface strains are principal strains, meaning that deformation is essentially governed by stretching. If deformation was due to vertical shear or included a large amount of through-thickness shear, the surface strains would not be principal strains [4].

As seen in Fig. 5, the strains at the end of the first stage are already located above the experimental FLC of the aluminium alloy AA1050-O. This is in close agreement with previous claims of the present authors that formability in SPIF is limited by fracture instead of necking [3, 4].

\subsection{Second stage}

The SPIF part at the end of the second stage can be produced in two different ways: first, DD corresponding to the first as well as the second stage with downward tool movement; second, DU corresponding to the first stage with downward tool movement and the second stage with upward tool movement. Figure 6 presents the numerical and experimental values of strain on the surface of the parts for grid locations placed within the depth range $20-60 \mathrm{~mm}$, for the two different tool path combinations. As shown, the DD strategy presents a strain path close to plane strain conditions whereas the DU strategy presents a strain path that is moved towards biaxial strains. The overall agreement between measured and computed values is very good and the results in Fig. 6(b) show that strain paths differ and become

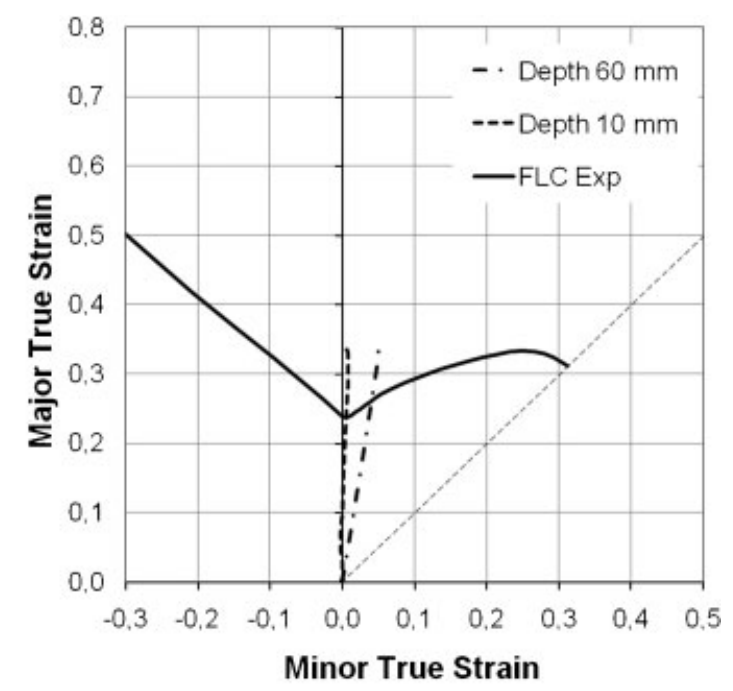

(b)

Fig. 5 First SPIF stage: (a) experimental and numerical strains at the end of the stage; (b) strain paths computed from two elements located at depths of $10 \mathrm{~mm}$ and $60 \mathrm{~mm}$ 


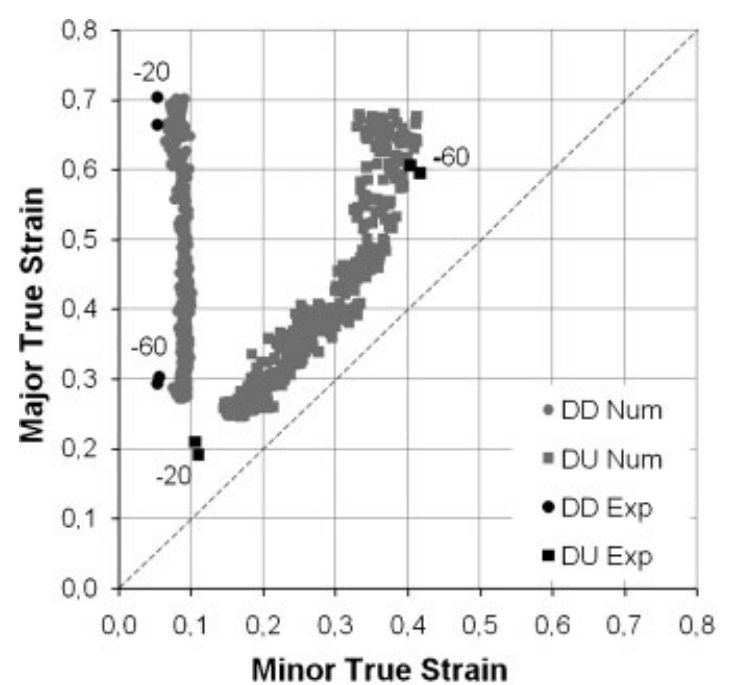

(a)

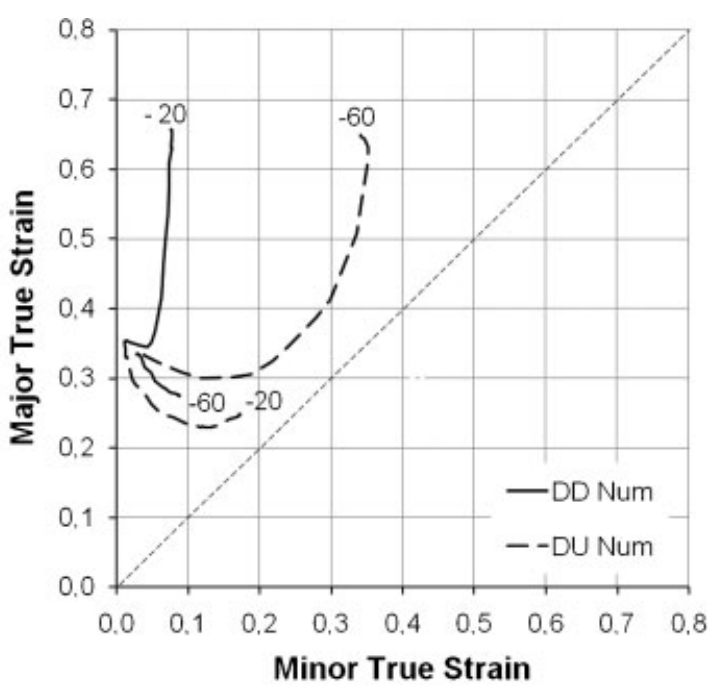

(b)

Fig. 6 Second SPIF stage: (a) experimental and numerical strains for DD and DU multi-stage SPIF strategies, where the enclosed strain values are computed within the depth range 20$60 \mathrm{~mm}$; (b) strain paths computed from two elements located within the depth range 20$60 \mathrm{~mm}$

highly non-linear in the second SPIF stage. This seems to be related to the decreasing strain in the $\varepsilon_{1}$ direction at the beginning of the second stage, corresponding to drawing rather than stretching deformation. The phenomenon is more pronounced in the SPIF parts produced by means of the DU forming strategy.

Figure 7 shows the distribution of thickness as a function of depth for DU and DD multi-stage SPIF strategies together with the predicted shape of the parts at the end of the second stage obtained from finite element modelling. The DD strategy causes a distribution similar to a conventional single-stage SPIF where increasing angle causes decreasing thickness. Using the DU strategy this is not the case, and most of the reduction in thickness occurs in the centre part where the drawing angle is smaller. This is necessary if vertical sides are to be achieved in the subsequent stages.

As seen, the shapes of the two parts differ considerably and, in the case of multi-stage SPIF with a DD strategy, a residual cone is found to

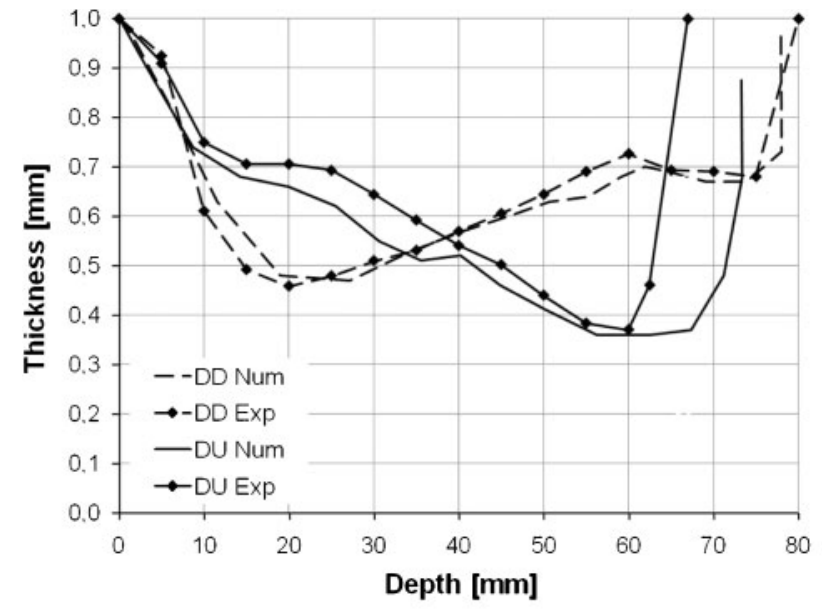

(a)

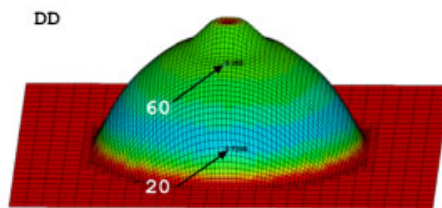

$1.000 \mathrm{e}+00$ $9.300 \mathrm{e}-01$ $8.600 \mathrm{e}-01$ $7.900 e-01$ $7.200 e-01$ $6.500 e-01$ $5.800 \mathrm{e}-01$

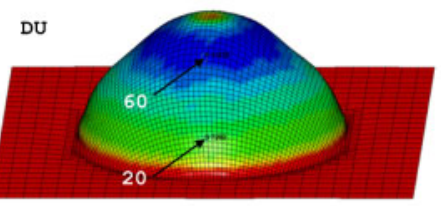
$5.100 \mathrm{e}-01$ $4.400 \mathrm{e}-01$ $3.700 \mathrm{e}-01$ $3.000 \mathrm{e}-01$

(b)

Fig. 7 Second SPIF stage: (a) experimental and numerical evolution of thickness as functions of depth for DD and DU strategies; (b) final shapes for DD and DU strategies obtained from finite element modelling (scale in millimetres) 
appear at the bottom end of the part. The residual cone is formed because the depth of the part is increased in the second stage, whereas the tool path only goes down by $70 \mathrm{~mm}$ in the first stage. Therefore, as the tool moves downwards during the second stage, a small plateau is formed beneath it (Fig. 8(a)). This plateau is observed experimentally as well as in the simulation. The existence of a residual cone has also been reported by Kitazawa and Nakane [6] in their original work on multi-stage SPIF.

In the case of multi-stage SPIF with a DU strategy, no residual cone is observed after the second stage. However, as shown in Fig. 8(b), material builds up in front of the tool and changes the contact condition between the tool and deforming part from point to linear. Again this phenomenon is observed in both experiments and simulations. The linear type of contact causes process forces in the $X-Y$ plane to increase and special care should be taken not to exceed the force limits of the machine when forming

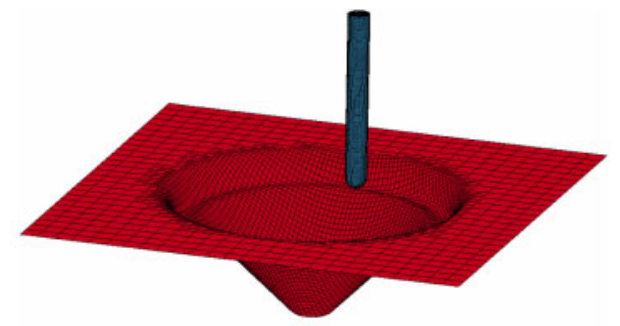

(a)

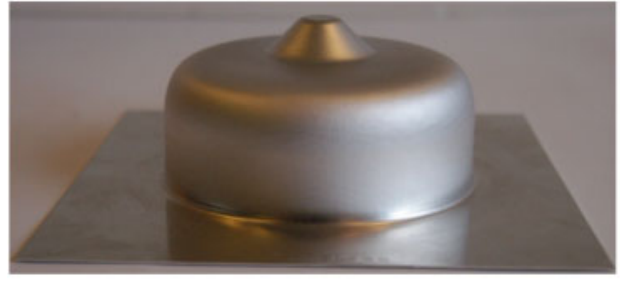

(c) harder materials. A similar result was found in experimental work performed by Kitazawa $e t$ al. [5].

However, neither of the aforementioned strategies utilized in the second stage succeeded in the production of flat-bottom cylindrical cups with vertical walls at the end of the multi-stage SPIF sequence (Figs 8(c) and (d)). The following section presents the solution-focused enhanced multi-stage SPIF sequence to solve this problem.

\subsection{Multi-stage sequence}

The multi-stage SPIF sequence that allows the production of a sound cylindrical cup with vertical walls and a residual cone at the bottom end of the part consists of three stages with a downward movement of the tool followed by a fourth stage with an upward movement of the tool (DDDU). Figure 9 shows the results obtained from experimentation and finite element analysis, and special emphasis is placed on the residual cone that

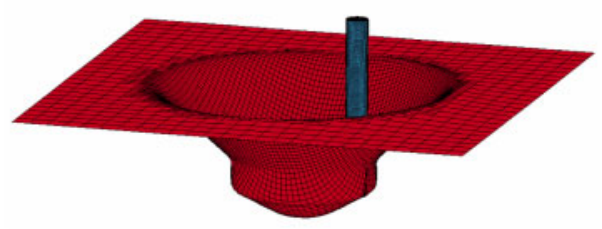

(b)

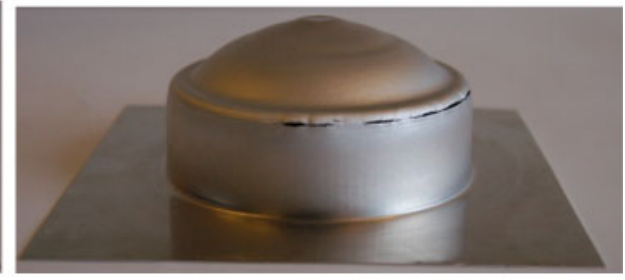

(d)

Fig. 8 Multi-stage SPIF of a cylindrical cup: (a) formation of a plateau during the second stage (DD strategy) obtained from finite element modelling; (b) formation of a linear contact during the second stage (DU strategy) obtained from finite element modelling; (c) final part obtained from a multi-stage DDDU strategy; (d) final part obtained from a multistage DUD(D) strategy
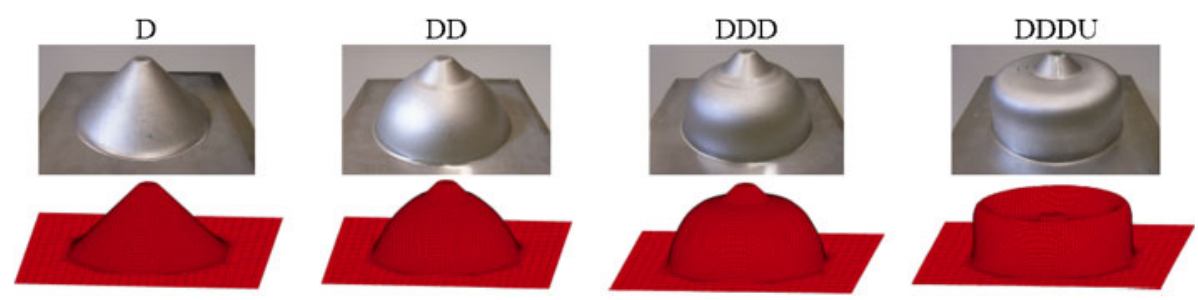

Fig. 9 Comparison between experimentation and simulation for each stage in the DDDU strategy 
develops immediately after the second stage and remains until the end of deformation. The overall agreement between experimentation and simulation is good during the first three initial stages but rather poor in the last stage. A possible explanation for this result is the too coarse mesh which is due to a large increase in surface area.

The elimination of the aforementioned residual cone requires enhancement of the multi-stage SPIF sequence with the aim of producing flat-bottom cylindrical cups with vertical walls (Fig. 10).

The enhanced multi-stage SPIF strategy proposed by the present authors makes use of the same number of stages and the same sequence as the previous DDDU strategy shown in Fig. 9. The shape resulting from the first stage is the same as the first step of the multi-stage SPIF strategy shown in Fig. 9. The second stage produces a deeper shape $(55 \mathrm{~mm})$ with a radius of curvature $R=10 \mathrm{~mm}$ at the bottom of the part in order to smooth the transition between the bottom and the conical wall. The third stage is designed to obtain a depth of $65.5 \mathrm{~mm}$ and the tool is forced to stop when it reaches the flat region located at the bottom centre of the part. The fourth stage is performed by upward movement of the tool starting at the bottom flat centre of the part located at a depth of $66.5 \mathrm{~mm}$. Table 2 provides the details.

\subsection{Formability limits}

Table 3 summarizes the multi-stage SPIF strategies that were analysed in the investigation. The forming stages, where cracking occurred, are indicated in parentheses and the experimental values of fracture
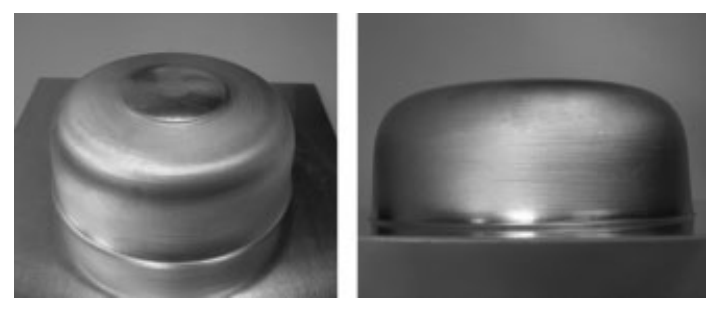

Fig. 10 Cylindrical cup obtained by means of the enhanced multi-stage SPIF strategy

Table 2 The depths of each stage that were utilized in the enhanced multi-stage SPIF strategy

\begin{tabular}{ll}
\hline Stage & Depth $(\mathrm{mm})$ \\
\hline $1(\mathrm{D})$ & 45 \\
2 (D) & 55 \\
3 (D) & 65.5 \\
$4(\mathrm{U})$ & 66.5 \\
\hline
\end{tabular}

Table 3 The different multi-stage SPIF strategies that were investigated

\begin{tabular}{ll}
\hline Strategy & Stages \\
\hline 1 & DDD(D) \\
2 & DDDU \\
3 & DUD(D) \\
4 & DUD(U) \\
5 & $-(D)-$ \\
\hline
\end{tabular}

strains were always measured for the forming stages that could not be completed because of fracture. The fracture strains were measured using the circle grid technique because no necking was found to occur (see section 2.1).

It is worth noting that strategy 5 results from the attempt to shape the geometry of the third stage directly from the initial sheet blank without resorting to intermediate forming stages. The outcome was the occurrence of fracture and a clear indication that multi-stage SPIF is advantageous over conventional SPIF because it is capable of distributing the strains more evenly throughout the sheet metal parts.

Figure 11 illustrates the distribution of thickness for the multi-stage SPIF strategies DDDU and DUD(D). As seen, the reduction in thickness using a DU strategy is slightly higher than that using a DD strategy. This is due to a shift in the strain field towards biaxial conditions which, for equal values of the principal strain $\varepsilon_{1}$, will necessarily lead to a higher reduction in thickness (see Figs 6 and 7). As a result of this, the DDDU strategy can be performed without fracture, whereas the DUD(D) strategy results in fracture during the fourth stage just after finishing the vertical section of the part.

The fracture appears in a zone with high thickness strain. Thickness measurements below this point are taken for only the first three stages because the fourth stage could not be completed. Both strategies give minimum thickness in the bending section between the vertical and the horizontal regions of the SPIF parts. This corresponds well to the theoretical strains which indicate a maximum thickness strain at the corner of the cups (see section 2.2). Using the suggested strategy it seems that the critical area is not the vertical sides themselves but the transition zone between vertical and horizontal. The reason is that this zone experiences a deformation close to equal biaxial stretching.

Figure 12 contains two different FFLCs. The thick solid line $\left(\varepsilon_{1}+1.08 \varepsilon_{2}=1.77\right)$ is obtained from the fracture strains measured in the experimental tensile and biaxial hydraulic bulge tests (see section 2). The dashed line is derived from the critical value of damage at the onset of cracking, and its slope is 


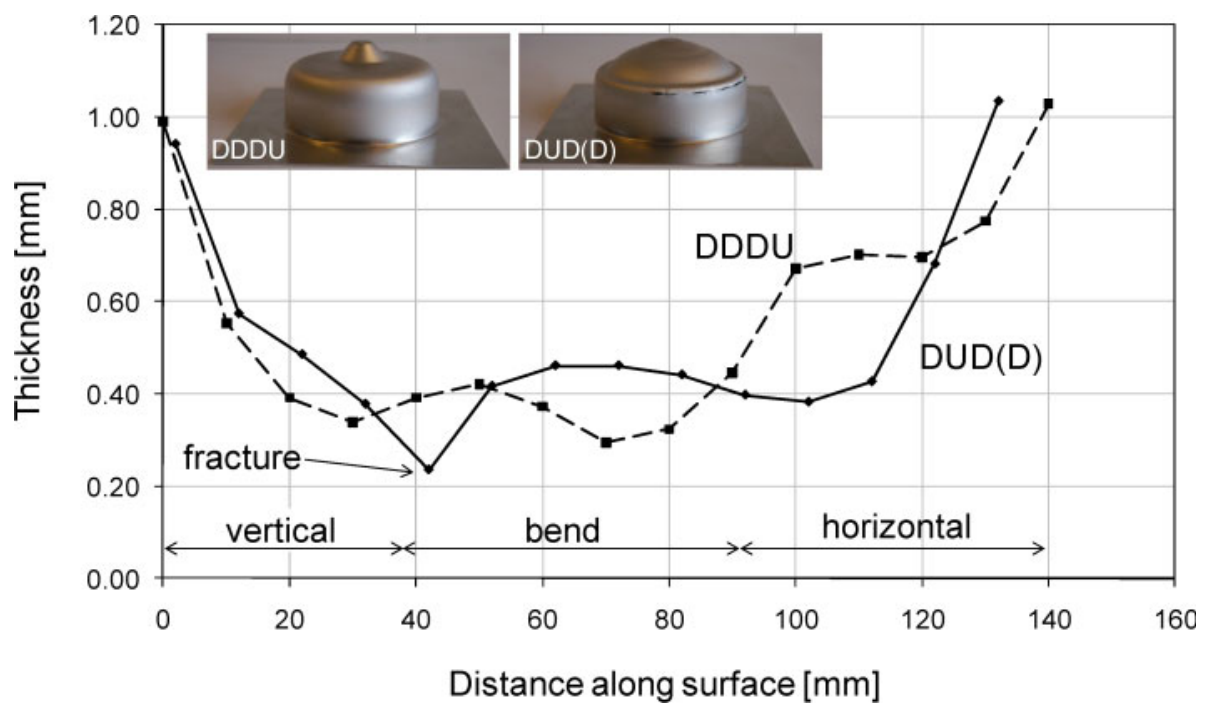

Fig. 11 Measured thickness of the multi-stage SPIF parts produced with DDDU and DUD(D) strategies as a function of the distance along the surface

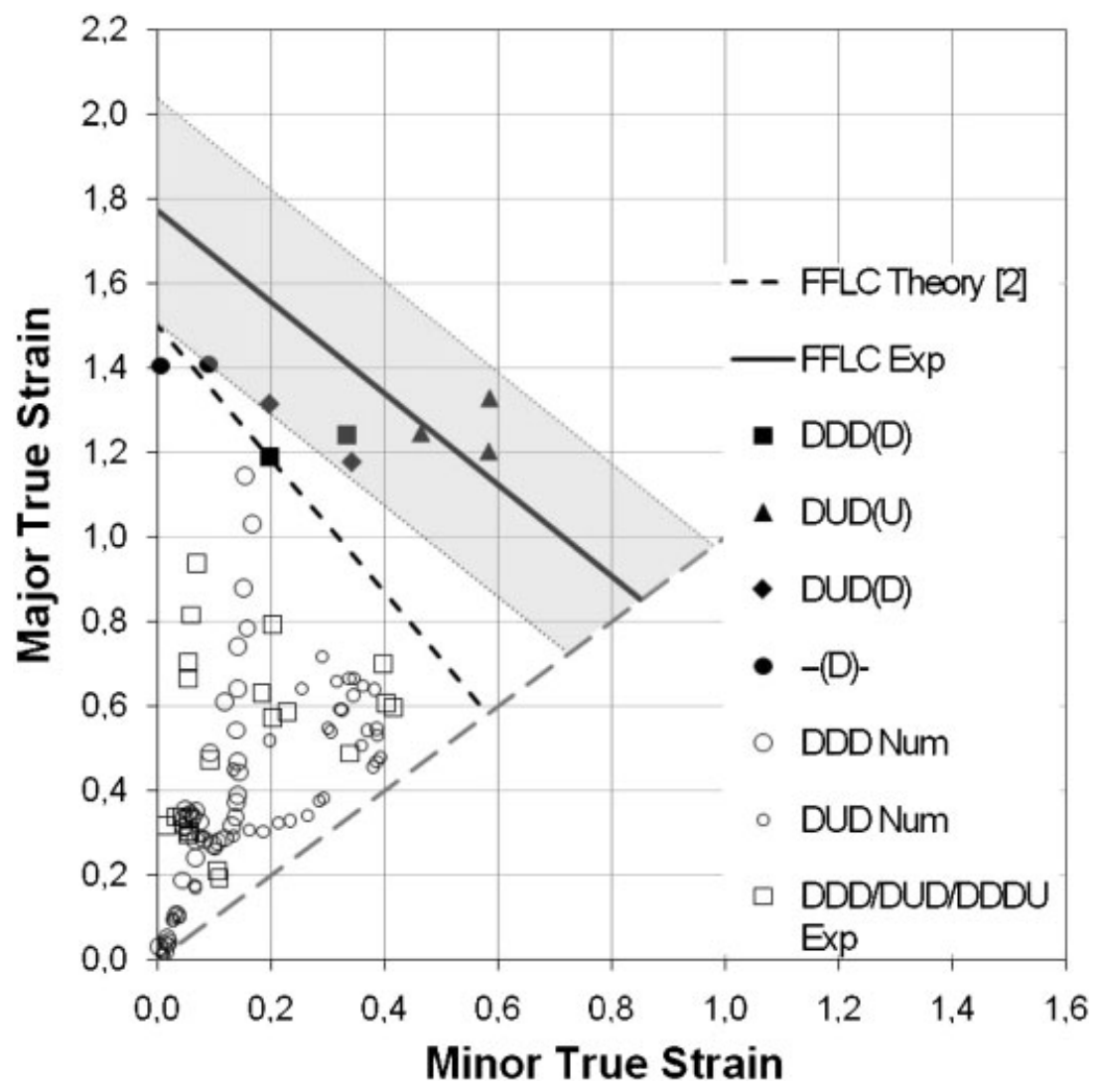

Fig. 12 Fracture forming-limit diagram containing the FFLCs and the strain values obtained for different multi-stage SPIF strategies. Filled symbols indicate data measured at fracture locations whereas open symbols indicate data measured and computed along the meridional section slice in order to cover the whole geometry of the parts 
determined in accordance with previous work published by the present authors $[\mathbf{3}, \mathbf{4}]$, according to

$$
\begin{aligned}
\frac{\varepsilon_{1}^{\text {biaxial }}-\varepsilon_{1}^{\text {plane strain }}}{\varepsilon_{2}^{\text {biaxial }}-\varepsilon_{2}^{\text {plane strain }}} & =\frac{\Delta \varepsilon_{1}}{\Delta \varepsilon_{2}} \\
& =-\frac{5\left(r_{\text {tool }} / t\right)+2}{3\left(r_{\text {tool }} / t\right)+6}
\end{aligned}
$$

To determine the equation for the dashed line a truncated conical shape characterized by various drawing angles with depth was formed until fracture. The experimental value of the drawing angle at fracture is $77.5^{\circ}$, corresponding to a strain $\varepsilon_{1}=1.5$ and to a final thickness $t=0.22 \mathrm{~mm}$. By substituting the thickness and the radius of the tool $\left(r_{\text {tool }}=6 \mathrm{~mm}\right)$ in equation (5) and by taking into account the above-mentioned value of strain at fracture, the corresponding FFLC is given by $\varepsilon_{1}+1.58 \varepsilon_{2}=1.5$.

The numerical and experimental values of strain resulting from the entire set of multi-stage SPIF strategies (Table 3) are also plotted in the principal strain space (Fig. 12). The values of strains without fracture were measured as well as simulated in the first three stages (DDD and DUD) and only measured in the fourth stage. As seen, all the strain points corresponding to sound SPIF conditions (DDD, DUD and DDDU plotted as open symbols) are located below the FFLCs. Moreover, the agreement between FFLCs and fracture points (DDD(D), $\operatorname{DUD}(\mathrm{U})$ and DUD(D) plotted as filled symbols) is also good. Major deviations are found in the comparisons with the experimental FFLC and can be attributed to the measuring procedure of the gauge length strains because it is difficult to execute whenever the thickness of the specimens is very small. This is the reason why a 15 per cent uncertainty interval is recommended to be plotted around the experimentally determined FFLC (see the grey area in Fig. 12).

The difference between the two FFLCs can also be explained by the fact that the experimental data (solid line) give a general fracture line constructed from different testing methods (i.e. bulge and tensile tests) whereas the theoretical FFLC (dashed line) is built upon an analytical framework that was specifically developed for SPIF and a strain point $\left(\varepsilon_{1}, \varepsilon_{2}\right)$ at failure that needs to be obtained from SPIF experiments [3]. The three failure points for the DUD(U) strategy seem to be outliers and this can be a result of the linear contact between tool and workpiece which is observed as the tool moves upwards (see section 4.2). In fact, the theoretical FFLC is derived under the assumption of a small contact area localized at the radius of the tool instead of a linear contact along the side of the tool.

\section{CONCLUSIONS}

A multi-stage strategy is presented which allows the formation of a cup with vertical walls. The movement of the tool (upwards or downwards) in multistage SPIF has a considerable effect on the thickness distribution and position of strain points in the principal strain space. Tool paths going upwards imply more biaxial strains than downward tool paths, which are closer to plane strain conditions. The strain paths are linear in the first stage and highly non-linear in the subsequent stages.

The FFLC independently determined from tensile and hydraulic bulge tests can be successfully utilized for establishing the formability limits of multi-stage SPIF provided that an uncertainty interval is taken into consideration in order to avoid errors attributed to the measurement of the gauge length strains. The correlation between the experimental FFLC and that resulting from the theoretical framework proposed by the present authors $[\mathbf{3}, \mathbf{4}]$ is good and supports the claim that SPIF is limited by fracture instead of necking. In fact, the overall level of strains achieved in multi-stage SPIF is much higher than the experimental values of necking currently found in conventional sheet metal forming.

Finally, an improved multi-stage SPIF strategy that allows the production of flat-bottom cylindrical cups with vertical walls was presented and details given about the overall forming sequence.

\section{ACKNOWLEDGEMENTS}

Maria Beatriz Silva and Paulo Martins would like to acknowledge the Fundação para a Ciência e a Tecnologia, Ministério da Ciência, Tecnologia e Ensino Superior, Portugal (Project PTDC/EMETME/64706/2006) for financial support.

(C) Authors 2010

\section{REFERENCES}

1 Jeswiet, J., Micari, F., Hirt, G., Bramley, A., Duflou, J., and Allwood, J. Asymmetric single point incremental forming of sheet metal. CIRP Ann. Mfg Technol., 2005, 54, 623-650.

2 Emmens, W. C. and van den Boogaard, A. H. Strain in shear and material behaviour in incre- 
mental forming. Key Engng Mater., 2007, 344, 519-526.

3 Silva, M. B., Skjoedt, M., Atkins, A. G., Bay, N., and Martins, P. A. F. Single-point incremental forming and formability-failure diagrams. J. Strain Analysis, 2008, 43(1), 15-35. DOI: 10.1243/03093247JSA340.

4 Silva, M. B., Skjoedt, M., Bay, N., and Martins, P. A. F. Revisiting single-point incremental forming and formability-failure diagrams by means of finite elements and experimentation. J. Strain Analysis, 2009, 44(4), 221-234. DOI: 10.1243/03093247JSA522.

5 Kitazawa, K., Wakabayashi, A., Murata, K., and Yaejima, K. Metal-flow phenomena in computerized numerically controlled incremental stretchexpanding of aluminum sheets (in Japanese). J. Japan Inst. Light Metals, 1996, 46, 65-70.

6 Kitazawa, K. and Nakane, M. Hemi-ellipsoidal stretch expanding of aluminum sheet by CNC incremental forming process with two path method (in Japanese). J. Japan Inst. Light Metals, 1997, 47, 440-445.

7 Kim, T. J. and Yang, D. Y. Improvement of formability for the incremental sheet metal forming process. Int. J. Mech. Sci., 2000, 42, 1271-1286.

8 Young, D. and Jeswiet, J. Wall thickness variations in single point incremental forming. J. Engng Mf., 2003, 217, 1571-1581.

9 Hirt, G., Ames, J., Bambach, M., and Kopp, R. Forming strategies and process modelling for CNC incremental sheet forming. CIRP Ann. Mfg Technol., 2004, 52(1), 203-206.

10 Bambach, M., Hirt, G., and Ames, J. Modelling of optimization strategies in the incremental $\mathrm{CNC}$ sheet metal forming process. In Materials processing and design: modeling, simulation and applications, Proceedings of the Eighth International Conference on Numerical methods in industrial forming processes (Numiform 2004), AIP Conference Proceedings, Vol. 712, Columbus, Ohio, USA, 13-17 June 2004, pp. 1969-1974 (American Institute of Physics, Melville, New York).

11 Skjoedt, M., Bay, N., Endelt, B., and Ingarao, G. Multi stage strategies for single point incremental forming of a cup. In Proceedings of the 11th Conference on Material forming (ESAFORM 2008), Lyon, France, 23-25 April 2008, paper 177 (Institut National des Sciences Appliquées de Lyon, Lyon).

12 Verbert, J., Belkassem, B., Henrard, C., Habraken, A. M., Gu, J., Sol, H., Lauwers, B., and Duflou, J. R. Multi-step toolpath approach to overcome forming limitations in single point incremental forming. In Proceedings of the 11th Conference on Material forming (ESAFORM 2008), Lyon, France, 23-25 April 2008, paper 587 (Institut National des Sciences Appliquées de Lyon, Lyon).
13 Duflou, J. R., Verbert, J., Belkassem, B., Gu, J., Sol, H., Henrard, C., and Habraken, A. M. Process window enhancement for single point incremental forming through multi-step toolpaths. CIRP Ann. Mfg Technol., 2008, 57, 253-256.

14 NP EN 10002-1 Metallic materials: tensile testing. Part 1: method of test (at room temperature), 1990 (Instituto Português da Qualidade, Caparica).

15 Rossard, C. Mise en forme des métaux et alliages, 1976 (Centre National de la Recherche Scientifique, Paris).

16 Atkins, A. G. Fracture mechanics and metal forming: damage mechanics and the local approach of yesterday and today. In Fracture research in retrospect (Ed. H. P. Rossmanith), 1997, pp. 327-350 (A.A. Balkema, Rotterdam).

17 Skjoedt, M., Silva, M. B., Martins, P. A. F., and Bay, N. Strain paths and fracture in multi stage single point incremental forming. In Proceedings of the Ninth International Conference on The technology of plasticity (ICTP 2008), Gyeongju, Republic of Korea, 7-11 September 2008, pp. 239-244 (Korean Society for Technology of Plasticity).

18 Skjoedt, M., Hancock, M. H., and Bay, N. Creating helical tool paths for single point incremental forming. Key Engng Mater., 2007, 334, 583-590.

\section{APPENDIX}

\section{Notation}

A

E

$r_{\text {tool }}$

$t$

$t_{0}$

$U_{\mathrm{T}}$ elongation at break point

Young's modulus

radius of the tool

thickness of the sheet

initial thickness of the sheet

toughness

meridional strain

circumferential strain

thickness strain

effective strain

half the cone angle of the component draw angle between the inclined wall and the initial flat configuration of the sheet

yield strength

effective stress 